\title{
Estilos de liderazgo en las redacciones chilenas: estudio de la autopercepción de los directivos ${ }^{1}$
}

\author{
Leadership styles in the chilean newsrooms: study of \\ managers' self-perception
}

\author{
Estilos de liderança nas salas de redação chilenas: estudo da \\ autopercepção dos gerentes
}

CRISTÓBAL BENAVIDES, Universidad de los Andes, Santiago, Chile [cbenavides@uandes.cl)

\section{RESUMEN}

Los cambios tecnológicos, la reducción de las barreras de entrada y la mayor competencia internacional han provocado una serie de trastornos en la industria de medios, que experimenta constantes transformaciones, además de la dificultad de encontrar un modelo de negocio rentable. El liderazgo, el trabajo en equipo, la creatividad y la innovación surgen como respuestas para enfrentar este escenario. Este estudio hace un levantamiento respecto de la autopercepción de liderazgo de los directores y editores generales de medios informativos chilenos. Con base en la aplicación del Multifactor Leadership Questionnaire (MLQ5x), se revelaron las principales características del liderazgo de acuerdo a la percepción de quienes dirigen las salas de redacción en medios nacionales. El carisma, la inspiración y la integridad son los rasgos más repetidos por los directores y editores, lo que los lleva a considerarse líderes transformacionales.

Palabras clave: liderazgo transformacional; medios de comunicación; salas de prensa; Chile; industria de comunicación.

\section{ABSTRACT}

Technological changes, reduced entry barriers and greater international competition have led to a series of disruptions in the media industry, which is experiencing a constant transformation, as well as dealing with the difficulty of finding a profitable business model. Leadership, teamwork, creativity and innovation arise as answer to face this scenario. This study surveys the self-perceived leadership of directors and general editors of Chilean media. Based on the application of the Multifactor Leadership Questionnaire (MLQ5x), we surveyed the main characteristics of leadership according to the perception of those who run the newsrooms in national media. Charisma, inspiration and integrity are the most repeated traits in the self-perception of directors and editors, leading them to see themselves as transformational leaders.

Keywords: transformational leadership; mass media; newsroom; Chile; Media industry.

\section{RESUMO}

As mudanças tecnológicas, a redução das barreiras de entrada e uma maior concorrência internacional causaram uma série de mudanças na indústria da mídia, que experimenta transformações constantes, bem como a dificuldade de encontrar um modelo de negócio lucrativo. Liderança, trabalho em equipe, criatividade e inovação, aparecem como respostas para enfrentar esse cenário. Este estudo realiza um levantamento sobre a autopercepção da liderança de diretores e editores em geral da mídia chilena. Com base na aplicação do Multifactor Leadership Questionnaire (MLQ5x), relevaram-se as principais características da liderança, de acordo com a percepção dos que dirigem as salas de redação na mídia nacional. Carisma, inspiração e integridade são os traços mais repetidos pelos diretores e editores, o que os leva a se considerarem líderes transformacionais.

Palavras-chave: liderança transformacional; mídia; salas de imprensa; Chile; indústria de comunicação. 


\section{INTRODUCCIÓN}

Desde hace más de una década, la industria de medios sufre una transformación relevante. A la dificultad de encontrar un modelo de negocio sólido que permita rentabilizar las operaciones en formato digital, se le sumaron cambios en los patrones de consumo. El Digital News Report (Newman, Fletcher, Levy, \& Nielsen, 2016), elaborado por el Reuters Institute, afirma que en la mayoría de los 26 países estudiados existe una pérdida de empleos que se traduce en una ardua competencia por reducir costos y frenar la caída de los ingresos. Pareciera que se está viviendo la tormenta perfecta. Chile no ha escapado de esta situación y constantemente-desde hace ya varios años-se puede leer en la prensa acerca de las desvinculaciones que se producen en las empresas de medios.

Es que la aparición de competidores que provienen de fuera de esta industria ha golpeado con fuerza al negocio. La fuga de audiencia hacia nuevas plataformas ha influido en la caída de la publicidad, ejemplares y cantidad de abonados. Los datos de la IAB Chile (2016) (Interactive Advertising Bureau) y la Asociación Chilena de Agencias de Publicidad (2017) muestra cómo las plataformas digitales, como Facebook y Google, por nombrar las principales, han ganado terreno. Si en 2007 la inversión online total llegaba a un 1,46\%, el 2016 acumuló un 16,8\%. A nivel mundial, según datos entregados por el Pew Research Center, desde 2015 estas firmas acaparan 70\% del avisaje digital, desplazando a sus competidores a puestos secundarios (Mitchell and Holcom, 2016).

En este complejo escenario hacer lo mismo de ayer o algo levemente similar ya no es una fórmula para el éxito. Es común encontrarse con una alta rotación de ejecutivos que intentan dar con nuevos modelos de financiamiento (Dal Zotto \& Van Kranenburg, 2008). Así, algunos ejecutivos de canales de televisión chilenos no completan ni siquiera un año en sus cargos (Cooperativa, 2016) y otros le atribuyen a "la volatilidad" de la industria la dificultad para manejar sus organizaciones (Obilinovic, 2016)

La prensa escrita tampoco ha estado ajena a los problemas. Según datos de la Asociación Nacional de la Prensa (2016a), la lectoría de los diarios nacionales pagados ha disminuido en un 30\% entre el 2007 y el 2016. Mientras que las revistas semanales y quincenales lo hicieron en un 20,3\% (Asociación Nacional de la Prensa, 2016b). En el caso de las radios, ha habido cambios de propiedad tendientes a consolidar aquellas emisoras que pertenecen a conglomerados que aplican economías de escala para poder ser competitivas.

Dado lo anterior, estudiar el liderazgo en los medios de comunicación se presenta cada vez más como algo relevante. Los constantes cambios que sufre esta industria, sumados a la caída de las barreras de entrada, la digitalización y la democratización de los medios, plantea que las personas se convierten en el activo clave para el éxito económico y editorial de los medios, lo que permite sobrevivir en un mercado cada vez más dinámico y competitivo. Es así como las exigencias de liderazgo migraron hacia las habilidades blandas que, combinadas con la capacidad de motivar al equipo y la flexibilidad y agilidad para adaptarse a los cambios (Koryak, Mole, Lockett, Hayton, Ucbasaran, \& Hodgkinson, 2015), parecieran ser el camino para conseguir buenos resultados (Koccedil, 2011). A eso, en la actualidad, habría que agregarle la rectitud, valor fundamental para los medios informativos en la llamada era de la posverdad (Peters, 2017). Las noticias falsas desdibujan las líneas entre ficción y no ficción, desafiando las prácticas periodísticas e incrementando el número de profesionales que usan noticias simuladas para desafiar los límites de lo que es noticia y la forma en que esta se crea. (Berkowitz \& Schwartz, 2016).

Peters (2017) señala que este fenómeno comenzó para evitar la discusión pública en un ciclo informativo que no se detiene durante las 24 horas. Es en ese proceso de sobreabundancia en la que algunos medios se abanderan ideológicamente y distorsionan deliberadamente la verdad. Además, existen grupos de interés que utilizan el conocimiento analítico de datos para mejorar su apariencia llamando la atención con informaciones no verdaderas (Harsin, 2015). Chile no ha sido la excepción y en los últimos meses se han destapado casos tanto en la prensa escrita como en la televisión. El diario La Tercera se vio afectado con una serie de colaboraciones de su corresponsal en España, quien inventó una docena de artículos y entrevistas, siendo la más resonada la del expresidente español José Rodríguez Zapatero, quien cumple el rol de mediador de la situación venezolana (La Tercera, 2017). El caso más emblemático, sin embargo, fue el artículo de la Revista Sábado, de El Mercurio, que reveló las mentiras de una persona que aseguraba haber matado a 12 delincuentes en legítima defensa y que fue entrevistada en diversas ocasiones por canales, diarios y radios sin recibir ningún tipo de cuestionamiento: ni a su actuar, ni a los datos y hechos descritos (Fluxá \& Galarce, 2017). 
Por esa razón, el liderazgo en las salas de redacciones toma relevancia debido a los retos a los que se enfrenta la industria. Se requieren directores y editores íntegros, con altos estándares profesionales, que guíen a sus equipos, los motiven y busquen siempre reflejar la verdad sin caer en cantos de sirena, los que les permitirán mejorar sus números a corto plazo, pero a la larga terminarán dañando la marca e imagen de sus empresas.

El propósito de este trabajo, que es la primera fase de un proyecto de investigación, es conocer la autopercepción de directores y editores de medios informativos chilenos respecto de sus características de liderazgo. Saber cuáles son esas características, descubrir qué estilo de liderazgo desarrollan y saber si están o no en línea con las innovaciones necesarias que exige la industria para poder mantener sus productos vigentes. En una futura segunda etapa, se estudiará a los periodistas y otros profesionales al interior de las salas de redacción para poder contrastarlos con estos resultados.

\section{LIDERAZGO TRANSFORMACIONAL: INNOVANDO A FAVOR DE LA ORGANIZACIÓN}

En toda compañía, incluidos los medios, el laborioso camino del liderazgo de personas ha pasado a ser un elemento clave (Christensen, Allworth, \& Dillon, 2012) para obtener ventajas competitivas (Jaiswal \& Dhar, 2015) en un mercado saturado de ofertas. Actores que vienen desde fuera, como Netflix y YouTube, cambiaron la forma de consumir contenido audiovisual. O Spotify y Apple Music, que modificaron la cadena de valor y el negocio de la música. Por esa razón, la industria de medios necesita de líderes que sean capaces de enfrentar esos desafíos y buscar nuevos negocios que les permitan rentabilizar sus productos y no solo depender de las suscripciones o de la venta de publicidad (WAN-IFRA, 2017).

Todas estas tareas van ligadas a la creación de ambientes creativos e innovadores (Johnson, Kompare, \& Santo, 2014). Gillet y Vandenberghe (2014) demostraron que el desarrollo de programas de liderazgo para motivar a los trabajadores contribuye a obtener rendimientos extraordinarios gracias a que influye positivamente en un mejor conocimiento y satisfacción laboral. Beam (2006) realizó un estudio a más de 1100 periodistas en Estados Unidos, en el que pudo determinar que los profesionales que se mostraban más satisfechos con su trabajo eran aquellos que consideraban que sus jefes valoraban el buen periodismo, las buenas prácticas y motivaban a sus equipos de trabajo, algo que en muchas partes se ha perdido por los recortes de costos que están sufriendo cientos de empresas periodísticas.

Distintos autores (Fairhurst \& Connaughton, 2014; Peters, 1987; Bennis, 1989; Kotter, 1982; Lynn, 1987; Vroom \&Jago, 2007; Yukl, 2002; Northouse, 2016) han listado las particularidades que deben poseer los líderes y que se pueden agrupar en las siguientes características: (1) tener una idea clara de qué es lo que quiere hacer; (2) reflejar pasión por lo que hacen y transmitir inspiración; (3) ser íntegro, sincero y confiable, pero también (4) curioso y audaz. Para Bass y Bass (2008), los líderes son personas diferentes, son individuos que ayudan a desarrollar entornos atractivos a los ojos de los trabajadores, proyectando mejoras, aunando motivaciones; así, logran que las personas generen resultados que marcan diferencias.

Una de las principales críticas que se escucha con frecuencia respecto de la falta de liderazgo en los medios de comunicación es la poca visión de largo plazo que posee gran parte de los directivos. Para muchos, la única obsesión pasa a ser el aumento en las ganancias, sin importar lo que suceda a futuro con las audiencias o la valoración de sus marcas. Además, está demostrado que las decisiones orientadas exclusivamente a obtener resultados inmediatos, sin pensar en la calidad, generan a la larga más daños que beneficios (Pérez-Latre \& Sánchez-Tabernero, 2012).

Durante los últimos años se han desarrollado diferentes clasificaciones de liderazgo, lo que dificulta poder delimitar el término a una definición (Northouse, 2016). Sin embargo, la mayoría de ellas comparten un punto de vista: el liderazgo implica un proceso de influencia y todos los líderes poseen uno o más seguidores (Vroom \& Jago, 2007). En la historia reciente, los académicos han dirigido sus estudios a diferenciar y clasificar los distintos estilos, enfoques o tipos de liderazgo, siendo el transformacional el que más ha concentrado la atención (Solà, i Badia, Hito, Osaba, \& García, 2016; Durán \& Castañeda, 2015).

Como se observa, los cambios que provocan los líderes en sus seguidores es la denominación en común que entregan los distintos autores. Para conseguirlo, aseguran que los líderes deben trabajar en generar altos grados de engagement (compromiso) y apelar a las motivaciones de largo plazo (Tansky \& Cohen, 2001). Como consecuencia, se genera una serie de actitudes positivas (Hu, Wayne, Bauer, Erdogan, \& Liden, 2016) que posibilitan resolver problemas y enfrentar nuevos desafíos con mayor apertura a la retroalimentación, 
enriqueciendo los procesos en todas las direcciones (Gillet \& Vandenberghe, 2014): jefes más dispuestos a escuchar y empleados más colaborativos.

Considerando las características diferenciadoras de la industria de comunicación, cuyos resultados son muy difíciles de predecir por sus altos grados de incertidumbre y volatilidad (Benavides, 2012; Picard, 2002), podemos ver que se trata de un sector en el que las personas son muy relevantes en el resultado final. Un análisis realizado por la consultora McKinsey (en Aris \& Bughin, 2012) se encargó de demostrar que la incertidumbre y la volatilidad del sector respecto de otras industrias es bastante mayor, es decir, el precio de un activo durante un tiempo determinado fluctúa mucho más que en otros sectores. A eso hay que sumarle que los ingresos tienden a ser cíclicos, porque el gasto de la inversión publicitaria, que es la principal y muchas veces la única fuente de financiamiento, está ligado al comportamiento de la economía.

Eso genera un alto nivel de vacilaciones que, combinado con los elevados costos de producción, representa el doble de riesgo que el que tiene, por ejemplo, la industria farmacéutica. Por eso, la correcta gestión del talento, el trabajo en equipo y la habilidad de los líderes para adaptarse a los cambios es un bien muy deseado en las organizaciones de medios (Küng, 2013).

En los últimos años, el liderazgo transformacional ha sido el estilo más estudiado (Wang, Oh, Courtright, \& Colbert, 2011). Se fundamenta en el proceso mediante el cual se cambia y transforma a los individuos que componen la organización. Se trata de un líder que logra que quienes trabajen con él dejen de pensar solamente en metas individuales y se sumen a las colectivas (Rubin, Munz, \& Bommer, 2005). Son profesionales que fijan metas de rendimiento exigentes pero alcanzables, con expectativas de que los resultados futuros serán mejores que los actuales (Jackson, Meyer, \& Wang, 2013).

Burns (1978) identificó el liderazgo transformacional como aquel líder que apela a las motivaciones intrínsecas para generar cambios en el equipo de trabajo, mientras que Bass (1985) lo describe desde los efectos que produce sobre sus seguidores, siendo la autoestima y la motivación las principales herramientas de cambio para conseguir altos estándares de desempeño.

Los principales componentes del liderazgo transformacional se pueden agrupar en cinco dimensiones: (1) el carisma, que corresponde al grado en el cual el líder se comporta de manera tal que provoca que los seguidores se identifiquen con él (Li \& Yuan, 2017); (2) la influencia idealizada (atribuida y conductual), que permite saber si el líder es percibido como confidente y poderoso, enfocado en ideales, creencias y ética de alto orden (Veríssimo \& Lacerda, 2015); (3) la motivación inspiracional, mecanismo mediante el cual el líder articula la visión y estimula a sus colaboradores (Sun $\&$ Henderson, 2017) con objetivos a futuro; (4) la estimulación intelectual, definida como un cuestionamiento a las acciones que tradicionalmente se realizan en la organización, es decir, a romper el statu quo, y (5) la consideración individualizada, es decir, que el líder trata a cada subordinado diferenciadamente, de acuerdo con sus necesidades y capacidades.

Tradicionalmente, el liderazgo transformacional se ha comparado con el liderazgo transaccional, en el que los líderes utilizan la negociación con los subordinados a cambio del logro de objetivos y metas organizacionales (recompensa contingente). Además, suelen monitorear muy de cerca las actividades de sus subordinados con el propósito de evitar posibles errores o desviaciones de los procedimientos y normas establecidos (dirección por excepción activa) (Bass \& Riggio, 2006).

Para lograr el rendimiento esperado, este tipo de líderes motiva a los subordinados a actuar como se espera, a diferencia del transformacional, que inspira a los seguidores a superar sus propias expectativas (Hermosilla, Amutio, da Costa, \& Páez, 2016) o del laissez -faire (ausencia de liderazgo), que se produce cuando los líderes evitan tomar decisiones y no están presentes para aclarar dudas o resolver problemas (Bass, 2008).

El liderazgo transformacional también está relacionado con el logro organizacional, el compromiso, la satisfacción y la confianza por parte de los subordinados, así como con su efectividad en equipos y organizaciones transformacionales, donde el valor de los resultados no solo es beneficioso para ellos, sino que para toda la organización (Men, 2014). Wang, Oh, Courtright, \& Colbert (2011) llegaron a la conclusión, después de analizar los últimos 25 años de investigación, de que el liderazgo transformacional tiende a ser un predictor robusto de resultados de rendimiento.

Se debe considerar que este tipo de liderazgo no es estático y se va moviendo de acuerdo a las necesidades que requiere la organización. Por lo tanto, se convierte en un estilo que puede ser de mucha utilidad en los medios de comunicación, que constantemente enfrentan cambios vertiginosos con nuevos actores que están modificando las reglas del consumo de información (Jenkins, Ford, \& Green, 2013).

Otra de las claves del liderazgo transformacional es que sabe anticiparse en las acciones -algo que quizá le 
ha faltado a los medios informativos tradicionales-, y explicar y fijar metas para que las personas se sientan valoradas por su inteligencia, creatividad y el aporte medible que hacen a la organización (Jackson, Meyer, \& Wang, 2013). Si a eso se suman los cambios en el mercado laboral, donde las personas buscan participar en proyectos en los que desarrollen su capacidad intelectual y tengan opciones de movilidad al interior de la compañía, la necesidad es aún mayor. Diferentes industrias ya han comenzado a desarrollar nuevas formas, más allá de las meramente económicas, para atraer personas (Garavan, O'Donnell, McGuire, \&Watson, 2007). Esta nueva visión del mundo del trabajo exige esfuerzos diferenciadores para adquirir, retener, motivar y desarrollar el talento (Bravo, Seibert, Kraimer, Wayne, $\&$ Liden, 2017) que necesitan las organizaciones para operar de manera eficiente y competitiva (Frank, Finnegan, \& Taylor, 2004). No es raro encontrarse con empresas que tienen dificultades para formar equipos de trabajo que satisfagan sus necesidades y reiteradas son las quejas de los ejecutivos respecto de una escasez y deterioro de las personas necesarias para conducir las divisiones más críticas de las organizaciones (Colbert, Barrick, \& Bradley, 2014).

El Washington Post es un buen ejemplo de lo que hay que hacer. Hace un par de años, creó el programa "La Red de Talento", que le permitió sumar más de dos mil periodistas alrededor del mundo, muchos de ellos freelance, otros retirados y algunos en ejercicio, pero con tiempo disponible (King \& Ockles, 2016). De esa manera, construyeron una potente red de colaboradores que les permite realizar coberturas noticiosas de calidad, prácticamente en cualquier parte del mundo.

Djibo, Desiderio y Price (2010) llegaron a la conclusión de que el comportamiento de los líderes transformacionales permite generar compromiso por parte de los trabajadores y reducir la cada vez más común alta rotación de personas, que genera gastos extras a las organizaciones. Chile no es la excepción. Mellado, Salinas, Valle y González (2010) lo confirmaron con un estudio realizado en cuatro grandes capitales regionales, incluida Santiago, para analizar el mercado laboral y el perfil del periodista. Las conclusiones arrojaron que siete de diez periodistas ha tenido entre dos y hasta quince trabajos diferentes en los últimos tres años, siendo los profesionales menores de 40 años los que presentan los más altos índices de movilidad.

Las nuevas condiciones laborales exigen poner énfasis en el desarrollo de habilidades de liderazgo al interior de las organizaciones e intentar llevar hacia niveles elevados el grado de compromiso. Para eso se debe apelar, principalmente, a las motivaciones intrínsecas que han sido identificadas como un importante conductor de creatividad e innovación en el trabajo (Oldham \& Cummings, 1996; Shin \& Zhou, 2003). Atmojo (2015) dejó en evidencia que el liderazgo transformacional influye significativamente en la satisfacción laboral y en el compromiso de la organización, la que a su vez tiene una influencia positiva en el rendimiento de los periodistas (Flores \& Subervi, 2014). Este círculo virtuoso (motivación, compromiso, satisfacción laboral) posibilitará la estimulación del aspecto intelectual abriendo espacios a procesos que conduzcan hacia nuevos conocimientos tanto en el ámbito tecnológico (Dougherty \& Hardy, 1996) como de formato y contenido.

La creatividad es uno de los primeros pasos para generar organizaciones innovadoras (Amabile, 1998) y su desarrollo es beneficioso para el éxito de la compañía (Henker, Sonnentag, \&Unger, 2015). En el mundo actual, las empresas deben aspirar a convertirse en entornos cada vez más creativos y sacar provecho de los beneficios que genera. De esa manera, la creatividad al interior de la empresa se debe ver como una apuesta a largo plazo y no como una forma rápida de encontrar soluciones a problemas actuales y específcos (Andriopoulos, 2001). Si antes solo se abordaban estos temas desde la perspectiva de la productividad, hoy se ha entendido la importancia de la creatividad como un factor necesario a desarrollar para poder cumplir consistentemente con los objetivos.

Gershon (2001) establece que los líderes de las empresas de medios deben crear procedimientos y políticas que soporten la implementación de la creatividad y la innovación como un pilar necesario para enfrentar escenarios ultra competitivos. Algo que Martin Baron (2016, p. 45), director del Washington Post, se encarga de confirmar: "Todos estamos trabajando duro, pero ahora debemos trabajar de manera más inteligente. La clave es la tecnología... si queremos ganar dinero, la innovación y creatividad en el área de los ingresos deberán coincidir con lo que observamos en la redacción".

Luego de estudiar en detalle la industria de TI en la India, Mittal y Dhar (2015) llegaron a la conclusión de que una de las formas más efectivas que tienen las empresas para conseguir sus ventajas competitivas depende del desarrollo de los procesos creativos, así como de la generación, retención y desarrollo de conocimiento en su interior. Para eso, es necesario que en la organización existan procesos formales en los que 
se comparta el conocimiento creativo, de modo que se sumen los esfuerzos por encontrar soluciones diferentes a los problemas cotidianos y esa forma de trabajar se convierta en un proceso reiterativo (Wang, 2010). Es tarea de los líderes animar a sus colaboradores a romper el statu quo y buscar rutas alternativas (Kark \&Van Dijk, 2007) duraderas para conectarse con una audiencia cada vez más esquiva.

El liderazgo transformacional asume de forma proactiva que, si se trabaja en encontrar soluciones creativas desde la cabeza de la compañía, todas las áreas y departamentos también lo harán. De esa manera, los directivos tendrán más opciones de conseguir resultados diferenciadores incluso en las tareas ordinarias (Mittal $\&$ Dhar, 2015). Este estilo de dirección ha demostrado ser la mejor forma de inspirar a los seguidores (Wang $\&$ Cheng, 2010) ya que, al adoptar un estilo transformacional, las personas pueden desarrollar y mejorar sus habilidades. Si esto se vuelve reiterativo, se pueden generar procesos que desemboquen en innovaciones relevantes.

Amabile (1998), con su modelo componencial, establece que la creatividad es el primer paso para la innovación. A su juicio, la creatividad surge de la interacción de una serie de elementos (entorno, experiencia, pensamiento creativo y motivación) que van influyendo en el comportamiento que tiene una persona a lo largo de su vida laboral. El primer impacto lo genera el entorno en el cual la persona se desenvuelve. Por esa razón, en entornos más competitivos hay más posibilidades de aumentar la creatividad. Un segundo factor que impacta la creatividad es la experiencia que tiene cada una de las personas. Todo aquello que un sujeto sabe y domina puede transformarlo en soluciones novedosas para la organización. Además, en la medida en que los individuos sienten que el líder los apoya y fomenta el conocimiento (Wang, 2010) la sensación de contribución es mayor.

Zhang y Bartol (2010) estudiaron en profundidad la relación de los empleados con sus superiores y dedujeron que mientras mayor es el nivel de involucramiento entre las personas, el líder y la organización en el proceso creativo, existen más posibilidades de obtener retornos por sobre el promedio. Por esa razón, independientemente del tipo de industria en la que se opere, la innovación se ha convertido en un elemento fundamental para conseguir resultados a largo plazo (Hon, 2012). Así, se cumple la máxima en los negocios: todo aquello que no crece, desaparece. Son la innovación y la creatividad, que están intrínsecamente unidas, las principales armas para combatir el fracaso (Robinson \& Beesley, 2010), porque sin cambios las empresas se van quedando atrás y hoy quedarse atrás puede ser fatal. Se requiere establecer nuevos procesos para crear y generar valor (Nieves, Quintana, \& Osorio, 2014).

El liderazgo transformacional está llamado a realizar esas tareas, ya que se ha demostrado que a mayor apoyo, mayor grado de innovación (Cerne, Jaklic, \& Škerlavaj, 2013). Estos cambios deben ir acompañados con estrategias y nuevos modelos de negocio que permitan crear y capturar valor (Afuah, 2014), desafiando lo establecido.

Aris y Bughin (2012) afirman que el desafío en la industria de medios para mantener a las organizaciones en la cima pasa por entender que se trata de un negocio de personas para personas, en el que los creadores del contenido son los encargados de crear el producto que se comercializa día tras día. Cuanto mejor sea el liderazgo que permita desarrollar al máximo las capacidades de cada uno de los miembros, mejor será el resultado que podrá obtener esa compañía. En esa línea, Sánchez-Tabernero (2008) asegura que los líderes deben trabajar conscientemente en la motivación de los subordinados para que estos desarrollen tareas útiles y atractivas. Baron (2016) lo reafirma al comentar que los periodistas siempre tienen algo significativo que los motiva, y es a ese sentimiento al que deben apelar los líderes para conducir sus equipos a crear productos exitosos.

\section{METODOLOGÍA}

El objetivo general del estudio es averiguar si los directores y editores de medios informativos chilenos se autoperciben como líderes transformacionales y si consideran que el actual escenario (cambios digitales y entrada de nuevos actores) hace necesario un nuevo estilo de liderazgo al interior de sus organizaciones.

Así, los objetivos específicos de este estudio son: (I) describir y conocer la autopercepción de los estilos de liderazgo que tienen los directores y editores de medios informativos chilenos; (II) determinar si en los medios chilenos existen o no rasgos distintivos del liderazgo transformacional (innovación, creación de valor y desarrollo de una cultura interna que promueve el talento y el trabajo en equipo); y (III) saber si la influencia de variables como tamaño, soporte, tipo de propiedad y años de la empresa son o no aspectos relevantes para la promoción de innovación y fomento de la creatividad (propiedades clave del liderazgo) dentro de estas instituciones. 
Para cumplir con el objetivo general de este trabajo, se aplicó un cuestionario estructurado que consta de tres partes y que permite conocer el comportamiento y los estilos de liderazgo. En la primera parte se utilizó el Multifactor Leadership Questionnaire 5X, comúnmente conocido como MLQ5x, que fue desarrollado por Avolio y Bass (2004). Este instrumento ha sido validado para diferentes países y actividades (Mittal \& Dhar, 2015; Qu, Janssen, \& Shi, 2015; Hartog, Mujen, \& Koopman, 1997; Pilbeam, Doherty, Davidson, \& Denyer, 2016; Zwingmann, Wegge, Wolf, Rudolf, Schmidt, \& Richter, 2014) y para algunos es considerado el mejor método para medir el liderazgo transformacional (Özaralli, 2003; Kirkbride, 2006) ${ }^{2}$

El MLQ5x es una herramienta multidimensional que posibilita la autoevaluación; en este caso, conocer cómo los directores y editores se autoperciben con respecto al estilo de liderazgo. Por medio de 45 enunciados, permite identificar y medir los aspectos del liderazgo transformacional mencionados anteriormente. Asimismo, posibilita que se reconozcan las características de un líder transformacional al incluir nueve ítems relacionados con la efectividad, la satisfacción de los empleados y el esfuerzo extra. El total de preguntas se agrupa en tres dimensiones: liderazgo transformacional, liderazgo transaccional y liderazgo correctivo, las que a su vez se dividen en nueve variables (véase la tabla 1). Todos los ítems fueron contestados usando una escala de Likert de cinco puntos, donde $0=$ totalmente en desacuerdo y 4 = totalmente de acuerdo.

Una vez finalizadas las preguntas del MLQ5x, los participantes debían contestar la segunda parte del cuestionario para cumplir con el segundo objetivo específico de este trabajo. Con el propósito de establecer la relación entre el liderazgo y la creatividad e innovación, se utilizó como base un instrumento desarrollado por Zhou y George (2001). Las preguntas fueron agrupadas en cinco ítems: motivación, innovación, audiencia, participación y talento. La tercera parte y final del cuestionario, que apunta al objetivo específico número tres de esta investigación, pretende establecer si las diferencias de tamaño, edad y propiedad de la organización tiene alguna relación con el liderazgo, la cultura de la innovación y la creatividad.

Para la aplicación de este cuestionario se usaron dos mecanismos. El primero fue el envío de un correo con el cuestionario de autoaplicación a una base de datos de 200 directores y editores de medios informativos (televisión, radio, diarios, revistas y medios digitales) de Santiago y regiones (Antofagasta, Iquique, Valparaíso, Concepción, Nuble, Araucanía y Los Lagos) durante los meses de septiembre, octubre y noviembre de 2016. Esta base de datos se construyó gracias a una estrategia mixta. Primero, se contactó a medios informativos con cobertura nacional y regional en Chile para obtener la dirección de los correos electrónicos de los posibles participantes. Posteriormente, se utilizó la técnica de bola de nieve (Bryman, 2012; Gilbert, 2008) que permite que las personas que aceptan colaborar entreguen datos de otros posibles directores y editores.

El correo electrónico fue remitido de manera individual para proteger la identidad y anonimato de cada uno de ellos. En el mensaje se les informó de la finalidad del estudio y sus objetivos. También se les aseguró

\begin{tabular}{|c|c|c|c|}
\hline Variables & Indicadores & Siglas & $\begin{array}{l}\text { Cantidad de ítems } \\
\text { en el cuestionario }\end{array}$ \\
\hline \multirow{5}{*}{ Liderazgo Transformacional } & 1. Influencia idealizada como atribuida & $\| A$ & \multirow{5}{*}{20} \\
\hline & 2. Influencia idealizada como conductual & IIC & \\
\hline & 3. Motivación inspiracional & Ml & \\
\hline & 4. Estimulación intelectual & $\mathrm{El}$ & \\
\hline & 5. Consideración individualizada & $\mathrm{Cl}$ & \\
\hline \multirow{2}{*}{ Liderazgo Transaccional } & 1. Recompensa contingente & $\mathrm{RC}$ & \multirow{2}{*}{9} \\
\hline & 2. Dirección por excepción activa & DPE-A & \\
\hline \multirow{2}{*}{ Liderazgo Laissez-faire } & 1. Dirección por excepción pasiva & DPE-P & \multirow{2}{*}{9} \\
\hline & 2. Laissez-faire & LF & \\
\hline
\end{tabular}

Tabla 1. Variables del MLQ

Fuente: Elaboración propia. 


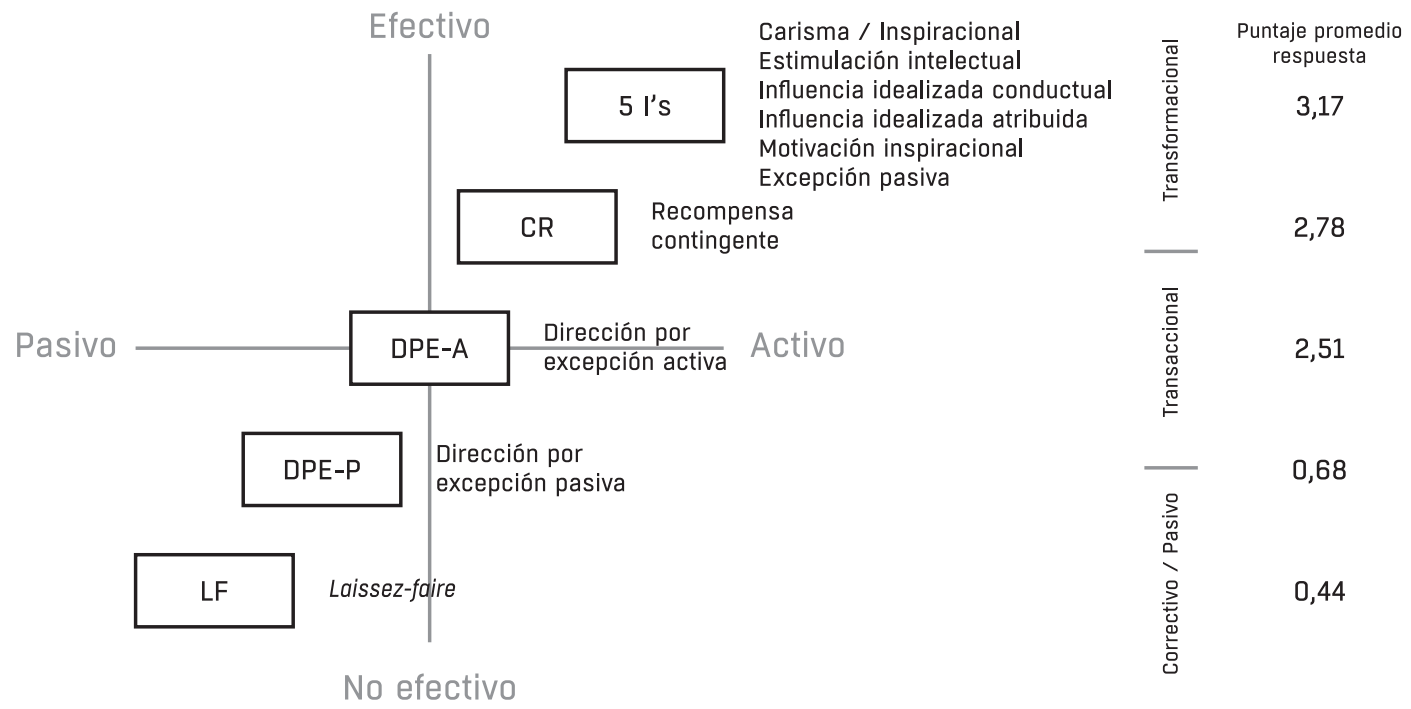

Figura 1. Snapshot de los estilos de liderazgo

Fuente: Elaboración propia.

la confidencialidad en el tratamiento de los datos, sin que sus respuestas pudieran ser identificadas de manera particular. Bajo esta modalidad, se lograron 12 respuestas.

Como segundo mecanismo y para aumentar el grado de participación, se visitaron medios en Santiago (de los cuatro soportes) con cobertura nacional y con mayor difusión en términos de audiencia y circulación, para que sus directores y editores contestaran cara a cara el cuestionario. Así, se lograron las 40 respuestas restantes, llegando a un n final de 52. De la totalidad de estas, 6 son directores y 46 editores. De ellos, 15 trabajan en canales de televisión, 26 en prensa escrita, 6 en radio y 5 en medios digitales.

\section{RESULTADOS Y COMENTARIOS}

Al analizar los resultados de la primera parte del cuestionario, se calculó la media (promedio) y la desviación estándar (DE) de las respuestas para conocer la autopercepción de liderazgo de los directores y editores de los medios que participaron en la muestra. La desviación estándar es el índice numérico que muestra la dispersión de un conjunto de datos; mientras más pequeña sea, mayor será la concentración de datos alrededor de la media. Esto quiere decir que se tuvo una mayor cantidad de respuestas con el mismo rango (Salkind, 1999).
La figura 1 refleja una fotografía (snapshot) de la autopercepción, en la que se observa que la mayoría de los participantes se define como un líder transformacional al obtener un puntaje promedio de 3,17. Esto quiere decir que los participantes contestaron con bastante frecuencia que se consideran líderes efectivos y activos en la relación con sus subordinados y respecto de la manera en la que gestionan sus salas de redacción.

De acuerdo a los resultados obtenidos (tabla 2), los directores y editores se consideran líderes transformacionales, siendo la motivación inspiracional (media: $3,29)$ el indicador con más alto promedio. Esto se puede interpretar como personas que creen que logran inspirar al resto, articulan de manera simple objetivos compartidos y comprenden lo que es correcto e importante, proporcionan visiones de lo que es posible y cómo alcanzar las metas, y realzan el significado y promueven expectativas de lo que hay que hacer (Avolio \& Bass, 2004). Todos los anteriores son rasgos deseables en la industria de medios, donde los trabajadores que están más satisfechos, motivados e inspirados con sus jefes logran mostrarse más complacidos con sus trabajos lo que, según la evidencia, se traduce en trabajadores más productivos (Czech \& Forward, 2013).

Otro de los indicadores que muestra un puntaje alto es la influencia idealizada conductual (media: 3,26), que responde a quienes actúan con integridad. Los puntajes altos en esta escala son típicos para los líderes que 


\begin{tabular}{lccc} 
Variable & Media & $\mathrm{DE}$ & Rango \\
\hline Influencia idealizada conductual & 3,26 & 0,87 & $0-4$ \\
\hline Influencia idealizada atribuida & 2,92 & 1,09 & $0-4$ \\
\hline Motivación inspiracional & 3,29 & 0,76 & $0-4$ \\
\hline Estimulación intelectual & 3,24 & 0,75 & $0-4$ \\
\hline Consideración individualizada & 3,18 & 0,9 & $0-4$ \\
\hline Recompensa contingente & 2,78 & 0,72 & $0-4$ \\
\hline Dirección por excepción activa & 2,51 & 1,04 & $0-4$ \\
\hline Dirección por excepción pasiva & 0,68 & 0,89 & $0-4$ \\
\hline Laissez-faire & 0,44 & 0,87 & $0-4$ \\
\hline
\end{tabular}

Tabla 2. Media y desviación estándar (DE)

Los valores son la media que se obtiene de una escala de tipo Likert de cinco puntos por elemento $(0=$ nunca; 1 = de vez en cuando; 2 = a veces; 3 = bastante; 4 = siempre $) n: 52$ para todas las respuestas.

Fuente: Elaboración propia.

muestran comportamientos positivos y altamente valorados, como el dominio, la conciencia, el autocontrol, el optimismo y la autoeficiencia (Avolio \& Bass, 2004). Hablan de sus valores y creencias más importantes y casi siempre consideran la moral y la ética. Iaoming, George y Cong (2010) demostraron que para los periodistas es muy importante el valor de la integridad para poder cumplir con su misión pública y comprometerse con los objetivos asignados a sus tareas. Estos tipos de líderes también se dedican a construir un sentido compartido de una visión y misión para el equipo.

Un tercer indicador que recibe una alta valoración es la estimulación intelectual (media: 3,24). Esta dimensión fomenta el pensamiento innovador, estimula las ideas y valores de las personas que trabajan con el líder, anima a cuestionar las formas de trabajo y procesos que pueden estar obsoletos o ser inapropiados para resolver un determinado problema (Avolio $\&$ Bass, 2004), fomentan la creatividad y la eficacia, y además son personas que estimulan intelectualmente al resto. Esta característica puede ser útil para los medios informativos que necesitan desafiar lo conocido y sumarse a las nuevas formas de consumo que demandan las audiencias (Jenkins, Ford, \& Green, 2013).

Las características menos autopercibidas por los editores son las relacionadas con la ausencia de liderazgo (laissez-faire y dirección por excepción pasiva) que corresponde al estilo de liderazgo que no asume las responsabilidades y deberes asignados (Skogstad, Einarsen, Torsheim, Aasland, \& Hetland, 2007). La media para estas respuestas fue de 0,68 y 0,44 , respectivamente. Es decir, los participantes de este estudio se autoperciben como líderes que siempre están presentes, toman decisiones, resuelven dudas y actúan para solucionar los problemas.

Al depurar los datos y comparar los resultados por soporte (véase la tabla 3) se encuentran algunas diferencias entre los indicadores analizados y la forma en la que los participantes del estudio autoperciben el liderazgo. En el caso de la prensa escrita (diarios y revistas) y de la radio, los profesionales consideran que su estilo se basa principalmente en la motivación inspiracional, es decir, aquellas personas que comunican apasionadamente una futura organización idealista que puede ser compartida (Hater \& Bass, 1988). Respecto de los directivos de televisión y web, se percibe un mayor reconocimiento de la influencia idealizada conductual, que es el elemento carismático del liderazgo transformacional. En él, los líderes se convierten en modelos a seguir, admirados y respetados por sus seguidores (Gregory Stone, Russell, \& Patterson, 2004). La influencia idealizada en el liderazgo también implica integridad en la forma de conducta ética y moral (Tracey \& Hinkin, 1998).

Para medir la consistencia de los indicadores de la segunda parte del cuestionario, se realizó un análisis 


\begin{tabular}{|c|c|c|c|c|c|c|c|c|}
\hline & Prensa & & TV & & Radio & & Web & \\
\hline & Media & $\mathrm{DE}$ & Media & $\mathrm{DE}$ & Media & $\mathrm{DE}$ & Media & $\mathrm{DE}$ \\
\hline $\begin{array}{l}\text { Influencia idealizada } \\
\text { conductual }\end{array}$ & 3,26 & 0,87 & 3,45 & 0,64 & 3,33 & 0,63 & 3,33 & 0,47 \\
\hline $\begin{array}{l}\text { Influencia idealizada } \\
\text { atribuida }\end{array}$ & 2,92 & 0,89 & 3,18 & 0,87 & 2,83 & 0,88 & 3,04 & 0,48 \\
\hline $\begin{array}{l}\text { Motivación } \\
\text { inspiracional }\end{array}$ & 3,29 & 0,79 & 3,31 & 1,04 & 3,46 & 0,6 & 2,92 & 0,91 \\
\hline $\begin{array}{l}\text { Estimulación } \\
\text { intelectual }\end{array}$ & 3,24 & 0,89 & 3,42 & 0,77 & 3,31 & 0,58 & 3 & 0,2 \\
\hline $\begin{array}{l}\text { Consideración } \\
\text { individualizada }\end{array}$ & 3,18 & 1,1 & 3,38 & 0,91 & 3,33 & 0,83 & 2,83 & 0,59 \\
\hline $\begin{array}{l}\text { Recompensa } \\
\text { contingente }\end{array}$ & 2,59 & 1,02 & 3,48 & 0,86 & 3,25 & 0,85 & 2,92 & 0,44 \\
\hline $\begin{array}{l}\text { Dirección por } \\
\text { excepción activa }\end{array}$ & 2,51 & 0,69 & 2,64 & 1,19 & 2,38 & 0,97 & 2 & 0,56 \\
\hline $\begin{array}{l}\text { Dirección por } \\
\text { excepción pasiva }\end{array}$ & 0,68 & 0,75 & 0,51 & 0,71 & 0,75 & 0,7 & 0,96 & 0,67 \\
\hline Laissez-faire & 0,44 & 0,76 & 0,38 & 0,79 & 0,25 & 0,44 & 0,83 & 0,75 \\
\hline
\end{tabular}

Tabla 3. Comparación de variables por soporte

Fuente: Elaboración propia.

\begin{tabular}{lcccc} 
Variable & Factor 1 & Factor 2 & Factor 3 & Unicidad \\
\hline Decisiones estratégicas & 0,6792 & 0,0249 & $-0,3004$ & 0,4479 \\
\hline Satisfacción de los trabajadores, valor de la marca & 0,5244 & $-0,1232$ & 0,0351 & 0,7086 \\
\hline Conocimiento de la audiencia & 0,6104 & 0,0480 & 0,0396 & 0,6236 \\
\hline Promover engagement & 0,8061 & 0,0931 & 0,1989 & 0,3019 \\
\hline Fijar objetivos de innovación & 0,0151 & 0,6274 & 0,2968 & 0,5181 \\
\hline Nuevas tecnologías & 0,0498 & 0,5889 & 0,0140 & 0,6505 \\
\hline Formación para enfrentar cambios & 0,0901 & 0,7182 & 0,0993 & 0,4662 \\
\hline Asumir riesgos & 0,2346 & 0,0681 & 0,6480 & 0,5205 \\
\hline Seleccionar y retener talento & $-0,1162$ & 0,3107 & 0,6282 & 0,4953 \\
\hline
\end{tabular}

Tabla 4. Análisis factorial exploratorio

Nota: Se calcularon las alphas de Cronbach para cada uno de los factores presentados en la tabla 4, p<0,05.

Fuente: Elaboración propia.

factorial exploratorio que se complementó con el cálculo de las alphas de Cronbach de cada uno de ellos. Así, se pudieron agrupar nueve de los 14 indicadores en tres factores (véase la tabla 4): el primero se refiere a la creación de valor orientada al público, es decir, cómo los líderes, mediante la rápida detección de las estrategias 


\begin{tabular}{|c|c|c|c|c|}
\hline \multirow[t]{2}{*}{ Tamaño } & Pequeña & Grande & Indistinto & \\
\hline & $21 \%$ & $8 \%$ & $71 \%$ & \\
\hline \multirow[t]{2}{*}{ Propiedad } & Familiar & Capital diversificado & Indistinto & \\
\hline & $2 \%$ & $26 \%$ & $72 \%$ & \\
\hline \multirow[t]{2}{*}{ Soporte } & Prensa Escrita & Tv (pago+abierta) & Online & Indistinto \\
\hline & $8 \%$ & $16 \%$ & $68 \%$ & $8 \%$ \\
\hline \multirow[t]{2}{*}{ Antigüedad } & Joven & Madura & Indistinto & \\
\hline & $26 \%$ & $6 \%$ & $68 \%$ & \\
\hline
\end{tabular}

Tabla 5. Tipo de empresa que favorece la innovación y la creatividad

Fuente: Elaboración propia.

de sus competidores, la medición de intangibles como marca, la satisfacción y las preferencias de la audiencia y la promoción del engagement, pueden influir en mejores desempeños. El segundo está directamente relacionado con la innovación, ya que agrupa variables como la propuesta de los líderes a sus empleados para obtener metas específicas de innovación y las recompensas que se reciben a cambio, muchas de ellas ligadas a las nuevas tecnologías, pero también a la formación permanente que posibilita ir mejorando la performance. El tercero se asocia al desarrollo de la cultura interna que posibilita que una organización siga siendo competitiva, ya que agrupa variables como la retención y detección de talento, pero también el impulso que los líderes les dan a los trabajadores para proponer ideas y asumir riesgos.

Respecto del objetivo número tres de esta investigación, que buscaba saber si la influencia de variables como tamaño, soporte, tipo de propiedad y años de la empresa son o no aspectos relevantes para la promoción de innovación y fomento de la creatividad dentro de estas instituciones, se pudo establecer que dicha relación no existe (tercera parte del cuestionario) (véase la tabla 5). Un $71 \%$ de los entrevistados cree que las pequeñas o grandes empresas pueden favorecer indistintamente la cultura de la innovación y de la creatividad. Algo similar sucede con el tipo de propiedad, donde $72 \%$ opina que no es relevante si la organización es familiar o tiene su propiedad diversificada para ser innovadora. Respecto del tipo de plataforma, $68 \%$ cree que la plataforma online es la que permite impulsar más la innovación y la creatividad. En cuanto a la antigüedad de la organización, $68 \%$ cree que la cantidad de años no importa para ser más creativos e innovadores, mientras que el mismo porcentaje considera que la antigüedad no es relevante para fomentar estos conceptos.

\section{CONCLUSIONES Y CONSIDERACIONES FINALES}

El liderazgo es un tema cada vez más relevante en el área del management y, por cierto, también en la gestión de medios, donde las buenas ideas llevadas a cabo con éxito se convierten en elementos que logran marcar la diferencia (Gershon, 2015).

Los resultados obtenidos en este trabajo permiten concluir que --si bien existe cierto grado de complacencia por parte de los editores y directores respecto de su autopercepción de liderazgo-en este primer catastro exploratorio con base empírica se consideran a sí mismos líderes transformadores, motivadores e innovadores, que logran que las personas que trabajan con ellos desarrollen sus talentos y confíen en ellos (Northouse, 2016), respondiendo así al objetivo general de este trabajo. De hecho, la principal característica autopercibida es la motivación inspiracional, que permite inspirar a sus equipos y luchar por metas comunes (Avolio y Bass, 2004). Tomando en cuenta el mercado laboral actual, en el que existe una alta rotación de personas, el lograr equipos consolidados y motivados puede ser una ventaja relevante para mantenerse competitivo.

Con base en la encuesta realizada, se podría afirmar que existe una concordancia entre la teoría y la práctica respecto del liderazgo transformacional. La teoría establece que permite la generación de trabajos colaborativos (Rubin et al., 2005) y en la práctica ellos se autoperciben como inspiracionales, conductuales 
e idealizadores, innovadores y promotores del trabajo en equipo, rasgos distintivos de este concepto, lo que cumple el segundo objetivo de este trabajo: determinar qué cualidades del liderazgo transformacional están presentes en los medios informativos chilenos.

Si bien no existen diferencias significativas entre soportes (televisión, radio, prensa escrita y soportes digitales), hay ciertas similitudes entre los directores y editores de prensa escrita y web por un lado, y de radio y televisión por otro. Quizá las características y exigencias propias de cada soporte los hacen autopercibirse, con respecto a su estilo de liderazgo, de manera semejante sobre lo que tienen que hacer, de cierta forma influenciados por los retos y amenazas cada día más comunes que debe enfrentar cada uno de ellos.

Respecto del objetivo número tres de esta investigación, los directores y editores consideran que ni el tamaño ni el tipo de propiedad son determinantes para que una organización favorezca la innovación y la creatividad, por lo tanto, todas están llamadas a realizar las transformaciones necesarias para poder mantenerse competitivas. Será tarea de quienes las dirijan llevar adelante esa empresa (Drucker, 2014).

En una futura segunda etapa de este trabajo de investigación, que se ha desarrollado gracias al apoyo de Conicyt a través de su programa Fondecyt $\mathrm{N}^{\circ} 11150020$, se podrá contrastar la autopercepción de liderazgo con la percepción que tienen las personas que trabajan con esos editores y directores de medios informativos. De esa manera, se comprobará si existe concordancia entre las percepciones de ambos grupos.

Para futuras investigaciones, la mayor colaboración de las empresas y una mayor disposición de profesionales dispuestos a participar de este tipo de estudios permitirán tener un panorama aún más exhaustivo de la realidad del liderazgo en la industria informativa de medios. También repetirla permitiría conocer la evolución en este tema, pudiendo compararse la autopercepción de los líderes de medios en diferentes periodos.

Asimismo, la triangulación de los resultados combinando métodos cuantitativos con técnicas cualitativas -como la observación participante o entrevistas en profundidad con los directores y editores de los medios informativos- podría ayudar a complementar los resultados obtenidos y a contrastar sus autopercepciones con una mejor descripción de su actuar con los trabajadores al interior de las salas de redacción de los medios informativos.

En próximos trabajos relacionados con el liderazgo y los medios informativos chilenos, se podrían plantear interrogantes tales como la importancia del liderazgo en la retención del talento para evitar lo que en mercados informativos más maduros se denomina la juniorization (Goodman \& Steyn, 2017), la que ha afectado la calidad en las salas de redacción. También puede ser interesante medir la relación entre el liderazgo y la satisfacción laboral, tomando en cuenta los desafíos actuales de los medios informativos tradicionales, que tienen que competir con nuevos actores en la industria y en los que la gestión del talento de las personas pasa a ser un rol clave (Christensen, Allworth, \& Dillon, 2012).

Otra arista interesante a explorar podría apuntar a comparar realidades desde la perspectiva del liderazgo y la autopercepción con otros países, para establecer si existen similitudes o diferencias respecto de los estilos de dirección, considerando que los retos y desafíos que deben enfrentar son prácticamente similares en todo el mundo. Lo que sí queda claro es que el tema de liderazgo y los medios es un asunto sobre el cual queda mucho por descubrir.

\section{NOTAS}

1. Este trabajo fue realizado con el apoyo de Conicyt mediante el proyecto Fondecyt № 11150020.

2. Sin embargo, existe otra corriente que opina lo contrario. Reid (2009, p. 5) asevera que "no debemos sorprendernos demasiado si alguna teoría es socavada por otros que mantienen opiniones agudamente opuestas", como la de Northouse (2016) quien afirma que el marco conceptual del liderazgo transformacional no es del todo sólido, porque sus componentes no son suficientemente distintivos para diferenciarlos de otras teorías de liderazgo. Mientras que Muenjohn y Armstrong (2008) consideran que si bien el MLQ permite identificar los distintos tipos de líderes, para ellos es el modelo de liderazgo completo (full range model) el más apropiado para identificar las variables que componen el liderazgo transformacional y transaccional. Se conforma por tres variables: actividad, efectividad y frecuencia, lo que, a juicio de estos autores, permite identificar mejor las características del liderazgo. Otros como Tourish (2013), son más extremos, ya que consideran que todos los atributos positivos del liderazgo transformacional "pueden volverse hacia el lado oscuro y convertirse en personas santas” (p. 4) que se miran a sí mismas, que concentran el poder y desarrollan un 
sentimiento de superioridad, todo lo contrario a lo deseado. Yukl (1999) hace su crítica desde el aspecto metodológico al plantear que, para mejorar la herramienta, se debiesen describir mejor los procesos explicativos, agregar algunos comportamientos, especificar mejor las condiciones limitantes (variables situacionales) y corregir el sesgo hacia las concepciones heroicas del liderazgo. Asimismo, se debería establecer con mayor certeza que el liderazgo transformacional, por medio de la confianza, posibilita el aumento del compromiso con la tarea y la autoeficacia. Hoyt y Blascovich (2003) tomaron ese desafío y con las mismas variables que Yukl consideró como insuficientes para probar la validez de la teoría, pudieron comprobar de manera concluyente que la confianza es vista como el ingrediente clave que Ilevó a los seguidores a realizar mejor sus tareas. Algo similar realizaron Hermosilla et al. (2016), quienes demostraron en un estudio realizado en el País Vasco que el liderazgo transformacional predice la motivación a trabajar extra, agregando el matiz de que la satisfacción tiene más peso que la eficacia en reforzar la motivación. Asimismo, pudieron comprobar que al reforzar la eficacia y la satisfacción indirectamente aumenta la motivación.

\section{REFERENCIAS}

Afuah, A. (2014). Business Model Innovation: concepts, analysis and cases. New York: Routledge.

Amabile, T. M. (1998). How to kill creativity (Vol. 87). Boston, MA: Harvard Business School Publishing

Andriopoulos, C. (2001). Determinants of organizational creativity: a literature review. Management decision, 39(10), 834-841. https://doi.org/10.1108/00251740110402328

Aris, A. \& Bughin, J. (2012). Managing Media Companies: Harnessing Creative Value. Chichester: Wiley \& Sons.

Asociación Chilena de Agencias de Publicidad. (2017). Inversión en Comunicaciones Publicitarias 2016 [Investment in Advertising Communications 2016]. Retrieved from http://www.achap.cl/wp-content/ uploads/2017/06/documento-inversion-publicitaria-2016-achap.pdf

Asociación Nacional de la Prensa (2016a). Boletín de circulación y Lectura Diarios [Newspaper circulation and reading bulletin]. Retrieved from http://www.achap.cl/wp-content/uploads/2017/06/boleti_ diarios2_semestre_2016.pdf

Asociación Nacional de la Prensa (2016b). Boletín de circulación y Lectura Revistas. [Magazine circulation and reading bulletin]. Retrieved from http://www.achap.cl/wp-content/uploads/2017/06/boleti_ revistas_semestre_2016.pdf

Atmojo, M. (2015). The influence of transformational leadership on job satisfaction, organizational commitment, and employee performance. International research journal of business studies, 5(2), 113 128. https://doi.org/10.21632/irjbs.5.2.113-128

Avolio, B. J. \& Bass, B. M. (2004). MLQ: Multifactor leadership questionnaire. Redwood City, CA: Mind Garden.

Baron, M. (2016). Discurso en el Festival Gabo [Discourse at the Gabo Festival]. Fundación Nuevo Periodismo Iberoamericano. Retrieved from http://www.fnpi.org/es/fnpi/video/discurso-de-martinbaron-en-la-ceremonia-de-los-premios-gabo-2016

Bass, B. M. (1985). Leadership and performance beyond expectations. New York: Free.

Bass, B. M. \& Bass, R. (2008). The Bass handbook of leadership: Theory, research, and managerial applications. New York: Simon and Schuster.

Bass, B. M. \& Riggio, R. E. (2006). Transformational leadership. Hove: Psychology Press.

Beam, R. A. (2006). Organizational goals and priorities and the job satisfaction of US. Journalists. Journalism \& Mass Communication Quarterly, 83(1), 169-185. https://doi.org/10.1177/107769900608300111

Benavides, C. (2012). Innovation and Leadership in the Media Industry. Pamplona: EUNSA.

Bennis, W. (1989). On Becoming a Leader. Reading, MA: Addison-Wesley. 
Berkowitz, D. \& Schwartz, D. A. (2016). Miley, CNN and The Onion: When fake news becomes realer than real. Journalism Practice, 10(1), 1-17. https://doi.org/10.1080/17512786.2015.1006933

Bravo, J., Seibert, S. E., Kraimer, M. L., Wayne, S. J., \& Liden, R. C. (2017). Measuring Career Orientations in the Era of the Boundaryless Career. Journal of Career Assessment, 25(3), 502-525. https://doi.org/10.1177/1069072715616107

Bryman, A. (2012). Social Research Methods. Oxford: Oxford University Press.

Burns, J. M. (1978). Leadership. New York: Harper \& Row.

Cerne, M., Jaklic, M., \& Škerlavaj, M. (2013). Authentic leadership, creativity, and innovation: A multilevel perspective. Leadership, 9(1), 63-85. https://doi.org/10.1177/1742715012455130

Christensen, C. M., Allworth, J., \& Dillon, K. (2012). How will you measure your life? New York: Harper Business.

Cooperativa (2016, September 30). Jaime De Aguirre dejó su cargo en Canal 13 [Jaime De Aguirre resigns to Canal 13]. Retrieved from https://www.cooperativa.cl/noticias/entretencion/television/canal-13/ jaime-de-aguirre-dejo-su-cargo-en-canal-13/2016-09-30/160528.html

Colbert, A. E., Barrick, M. R., \& Bradley, B. H. (2014). Personality and leadership composition in top management teams: Implications for organizational effectiveness. Personnel Psychology, 67(2), 351-387. https://doi.org/10.1111/peps.12036

Czech, K. \& Forward, G. L. (2013). Communication, Leadership, and Job Satisfaction: Perspectives on Supervisor-Subordinate Relationships. Studies in Media and Communication, 1(2), 11-24. https://doi.org/10.11114/smc.vli2.122

Christensen, C. M., Allworth, J., \& Dillon, K. (2012). How will you measure your life? New York: Harper Business.

Dal Zotto, C. \& Van Kranenburg, H. (Eds.). (2008). Management and innovation in the media industry. Cheltenham: Edward Elgar Publishing.

Djibo, I. J., Desiderio, K. P., \& Price, N. M. (2010). Examining the role of perceived leader behavior on temporary employees' organizational commitment and citizenship behavior. Human Resource Development Quarterly, 21(4), 321-342. https://doi.org/10.1002/hrdq.20049

Dougherty, D. \& Hardy, C. (1996). Sustained product innovation in large, mature organizations: Overcoming innovation-to-organization problems. Academy of Management Journal, 39(5), 1120-1153. https://doi.org/10.2307/256994

Drucker, P. (2014). Innovation and entrepreneurship. New York: Routledge.

Durán, M. G. \& Castañeda, D. I. (2015). Relación entre liderazgo transformacional y transaccional con la conducta de compartir conocimiento en dos empresas de servicios [Relationship between transformational and transactional leadership with knowledge-sharing behavior in two service companies]. Acta Colombiana de Psicología, 18(1), 135-147. https://doi.org/10.14718/ACP.2015.18.1.13

Fairhurst, G. T. \& Connaughton, S. L. (2014). Leadership: A communicative perspective. Leadership, 10(1), 7-35. https://doi.org/10.1177/1742715013509396

Flores, M. \& Subervi, F. (2014). Assessing the job satisfaction of US Latino journalists. Journalism Practice, 8(4), 454-468. https://doi.org/10.1080/17512786.2013.849847

Fluxá, R. \& Galarce, A. (2017, July 22). El justiciero imaginario [The imaginary vigilante]. Revista Sábado. Retrieved from http://impresa.elmercurio.com/Pages/SupplementDetail.aspx?dt=2017-0722\&SupplementID $=1 \&$ BodyID $=0$

Frank, F. D., Finnegan, R. P., \& Taylor, C. R. (2004). The race for talent: retaining and engaging workers in the 21st century. Human Resource Planning, 27(3), 12-25. Retrieved from http://citeseerx.ist.psu. edu/viewdoc/download?doi=10.1.1.514.4132\&rep=repl\&type $=$ pdf

Garavan, T. N., O’Donnell, D., McGuire, D., \& Watson, S. (2007). Exploring perspectives on human resource development: An introduction. Advances in Developing Human Resources, 9(1), 3-10. https://doi.org/10.1177/1523422306294342 
Gershon, R. (2001). Telecommunications Management. New York: Routledge.

Gershon, R. (2015). Digital media and innovation: Management and design strategies in communication. Los Ángeles: Sage Publications.

Gilbert, N. (2008). Researching Social Life. London: Sage.

Gillet, N. \& Vandenberghe, C. (2014). Transformational leadership and organizational commitment: The mediating role of job characteristics. Human Resource Development Quarterly, 25(3), 321-347. https://doi.org/10.1002/hrdq.21192

Goodman, R. \& Steyn, E. (Eds.) (2017). Global Journalism Education In the 21st Century. Challenges \& Innovations. Austin: Knight Center for Journalism in the Americas.

Gregory Stone, A., Russell, R. F., \& Patterson, K. (2004). Transformational versus servant leadership: A difference in leader focus. Leadership \& Organization Development Journal, 25(4), 349-361. https://doi.org/10.1108/01437730410538671

Harsin, J. (2015). Regimes of posttruth, postpolitics, and attention economies. Communication, Culture $\mathcal{E}$ Critique, 8(2), 327-333. https://doi.org/10.1111/cccr.12097

Hartog, D. N., Muijen, J. J., \& Koopman, P. L. (1997). Transactional versus transformational leadership: An analysis of the MLQ. Journal of occupational and organizational psychology, 70(1), 19-34. https://doi.org/10.1111/j.2044-8325.1997.tb00628.x

Hater, J. J. \& Bass, B. M. (1988). Superiors' evaluations and subordinates' perceptions of transformational and transactional leadership. Journal of Applied psychology, 73(4), 695-702. https://doi.org/10.1037/0021-9010.73.4.695

Henker, N., Sonnentag, S., \& Unger, D. (2015). Transformational leadership and employee creativity: the mediating role of promotion focus and creative process engagement. Journal of Business and Psychology, 30(2), 235-247. https://doi.org/10.1007/s10869-014-9348-7

Hermosilla, D., Amutio, A., da Costa, S., \& Páez, D. (2016). El Liderazgo transformacional en las organizaciones: variables mediadoras y consecuencias a largo plazo [Transformational leadership in organizations: mediating variables and long-term consequences]. Revista de Psicología del Trabajo y de las Organizaciones, 32(3), 135-143. https://doi.org/10.1016/j.rpto.2016.06.003

Hon, A. H. (2012). Shaping environments conductive to creativity: The role of intrinsic motivation. Cornell Hospitality Quarterly, 53(1), 53-64. https://doi.org/10.1177/1938965511424725

Hoyt, C. L. \& Blascovich, J. (2003). Transformational and transactional leadership in virtual and physical environments. Small Group Research, 34(6), 678-715. https://doi.org/10.1177/1046496403257527

Hu, J., Wayne, S. J., Bauer, T. N., Erdogan, B., \& Liden, R. C. (2016). Self and senior executive perceptions of fit and performance: A time-lagged examination of newly-hired executives. Human Relations, 69(6), 1259-1286. https://doi.org/10.1177/0018726715609108

IAB (2016). Gasto Publicitario en Chile [Advertising expenditure in Chile]. Retrieved from http://iabtrends.cl/2016/12/22/gasto-publicitario-en-chile-tendra-un-alza-de-un-2-para-el-2017/

Iaoming, H., George, Ch., \& Cong, S. (2010). Job satisfaction of journalists: Professional aspirations, newsroom culture and social context. Media Asia, 40(1), 73-84. https://doi.org/10.1080/01296612.2013.11689952

Jackson, T. A., Meyer, J. P., \& Wang, X. H. F. (2013). Leadership, Commitment, and Culture: A MetaAnalysis. Journal of Leadership \& Organizational Studies, 20(1), 84-106. https://doi.org/10.1177/1548051812466919

Jaiswal, N. K. \& Dhar, R. L. (2015). Transformational leadership, innovation climate, creative self-efficacy and employee creativity: A multilevel study. International Journal of Hospitality Management, 51, 30-41. https://doi.org/10.1016/j.jhhm.2015.07.002

Jenkins, H., Ford, S., \& Green, J. (2013). Spreadable media: Creating value and mediaing in a networked culture. New York: NYU Press. 
Johnson, D., Kompare, D., \& Santo, A. (2014). Making media work: cultures of management in the entertainment industries. New York: NYU Press.

Kark, R. \& Van Dijk, D. (2007). Motivation to lead, motivation to follow: The role of the self-regulatory focus in leadership processes. Academy of Management Review, 32(2), 500-528. https://doi.org/10.5465/AMR.2007.24351846

King, S. \& Ockles, C. (2016, May 25). Cómo contratan talento externo PwC y The Washington Post [How PwC and The Washington post hire outside talent]. Retrieved from http://www.elheraldo. hn/economia/dineroynegocios/963643-466/c\%C3\%B3mo-contratan-talento-externo-pwc-y-thewashington-post

Kirkbride, P. (2006). Developing transformational leaders: the full range leadership model in action. Industrial and commercial training, 38(1), 23-32. https://doi.org/10.1108/00197850610646016

Koccedil, H. (2011). The impact of managers' leadership behaviors on job satisfaction and performance of employees. African Journal of Business Management, 5(30), 11836-11843. Retrieved from https://www. researchgate.net/publication/271227512_The_impact_of_managers'_leadership_behaviors_on_job_ satisfaction_and_performance_of_employees

Koryak, O., Mole, K. F., Lockett, A., Hayton, J. C., Ucbasaran, D., \& Hodgkinson, G. P. (2015). Entrepreneurial leadership, capabilities and firm growth. International Small Business Journal, 33(1), 89-105. https://doi.org/10.1177/0266242614558315

Kotter, J. P. (1982). The General Managers. New York: Free Press.

Küng, L. (2013). Strategic management in the media: Theory to practice. London: Sage.

La Tercera (2017, July 27). Las entrevistas que no debimos publicar [The interviews that we should not publish]. Retrieved from http://www.latercera.com/noticia/las-entrevistas-no-debimos-publicar/

Li, J. \& Yuan, B. (2017). Both angel and devil: The suppressing effect of transformational leadership on proactive employee's career satisfaction. International Journal of Hospitality Management, 65, 59-70. https://doi.org/10.1016/j.ijhm.2017.06.008

Lynn, L. E. (1987). Managing Public Policy. Boston: Little Brown Foundations of Public Management Series.

Mellado, C., Salinas, P., Del Valle, C., \& González, G. (2010). Estudio comparativo de cuatro regiones: mercado laboral y perfil del periodista [A comparative study in four regions: Labor market and profile of Chilean journalist]. Cuadernos de información, (26), 45-64. Retrieved from https://doi.org/10.7764/cdi.26.11

Men, L. R. (2014). Strategic internal communication: transformational leadership, communication channels, and employee satisfaction. Management Communication Quarterly, 28(2). https://doi.org/10.1177/0893318914524536

Mitchel, A. \& Holcomb, J. (2016). State of the News Media 2016. Retrieved from http://assets.pewresearch. org/wp-content/uploads/sites/13/2016/06/30143308/state-of-the-news-media-report-2016-final.pdf

Mittal, S. \& Dhar, R. L. (2015). Transformational leadership and employee creativity: mediating role of creative self-efficacy and moderating role of knowledge sharing. Management Decision, 53(5), 894-910. https://doi.org/10.1108/MD-07-2014-0464

Muenjohn, N. \& Armstrong, A. (2008). Evaluating the structural validity of the multifactor leadership questionnaire (MLQ), capturing the leadership factors of transformational-transactional leadership. Contemporary Management Research, 4(1). https://doi.org/10.7903/cmr.704

Newman, N., Fletcher, R., Levy, D. A., \& Nielsen, R. K. (2016). Reuters Institute digital news report 2016. Oxford: University of Oxford, Reuters Institute for the Study of Journalism. Retrieved from http://reutersinstitute.politics.ox.ac.uk/sites/default/files/research/files/ Digital\%2520News\%2520Report\%25202016.pdf

Nieves, J., Quintana, A., \& Osorio, J. (2014). Knowledge-based resources and innovation in the hotel industry. International Journal of Hospitality Management, 38, 65-73.

https://doi.org/10.1016/j.ijhm.2014.01.001 
Northouse, P. (2016). Leadership Theory and Practice. 7 ed., New York: Sage.

Obilinovic. D. (2016, November 15). CHV cambia de director ejecutivo luego de complejos 17 meses [CHV changes CEO after 17 difficult months]. Retrieved from http://www.latercera.com/noticia/chvcambia-director-ejecutivo-luego-complejos-17-meses/

Oldham, G. R. \& Cummings, A. (1996). Employee creativity: Personal and contextual factors at work. Academy of management journal, 39(3), 607-634. https://doi.org/10.2307/256657

Özaralli, N. (2003). Effects of transformational leadership on empowerment and team effectiveness Leadership \& Organization Development Journal, 24(6), 335-344. https://doi.org/10.1108/01437730310494301

Pérez-Latre, F. J. \& Sánchez-Tabernero, A. (2012). Innovación en los medios: la ruta del cambio [Media innovation: the road to change]. Pamplona: EUNSA.

Peters, T. (1987). Thriving on chaos: Handbook for a managerial revolution. New York: Alfred. A. Knopf.

Peters, M. A. (2017). Education in a post-truth world. Educational Philosophy and Theory, 49(6), 563-566. https://doi.org/10.1080/00131857.2016.1264114

Picard, R. G. (2002). The economics and financing of media companies (No. 1). New York: Fordham University Press.

Pilbeam, C., Doherty, N., Davidson, R., \& Denyer, D. (2016). Safety leadership practices for organizational safety compliance: Developing a research agenda from a review of the literature. Safety science, 86, 110-121. https://doi.org/10.1016/j.ssci.2016.02.015

Qu, R., Janssen, O., \& Shi, K. (2015). Transformational leadership and follower creativity: The mediating role of follower relational identification and the moderating role of leader creativity expectations. The Leadership Quarterly, 26(2), 286-299. https://doi.org/10.1016/j.leaqua.2014.12.004

Reid, M. (2009). A critique of 'transformational leadership 'theory. MBA paper. Retrieved from http://www.academia.edu/300040/A_critique_of_Transformational_Leadership_theory

Robinson, R. N. \& Beesley, L. G. (2010). Linkages between creativity and intention to quit: An occupational study of chefs. Tourism Management, 31(6), 765-776. https://doi.org/10.1016/j.tourman.2009.08.003

Rubin, R. S., Munz, D. C., \& Bommer, W. H. (2005). Leading from within: The effects of emotion recognition and personality on transformational leadership behavior. Academy of Management Journal, 48(5), 845-858. https://doi.org/10.5465/AMJ.2005.18803926

Salkind, N. (1999). Métodos de Investigación [Research methods]. Barcelona: Pearson Educación.

Sánchez-Tabernero, A. (2008). Los contenidos de los medios de comunicación: calidad, rentabilidady competencia [Media content: quality, profitability and competition]. Barcelona: Ediciones Deusto.

Shin, S. J. \& Zhou, J. (2003). Transformational leadership, conservation, and creativity: Evidence from Korea. Academy of management Journal, 46(6), 703-714. https://doi.org/10.2307/30040662

Skogstad, A., Einarsen, S., Torsheim, T., Aasland, M. S., \& Hetland, H. (2007). The destructiveness of laissez-faire leadership behavior. Journal of occupational health psychology, 12(1), 80-92. https://doi.org/10.1037/1076-8998.12.1.80

Solà, G. J., i Badia, J. G., Hito, P. D., Osaba, M. A. C., \& García, J. L. D. V. (2016). Self-perception of leadership styles and behaviour in primary health care. BMC health services research, 16(1), 572. https://doi.org/10.1186/s12913-016-1819-2

Sun, R. \& Henderson, A. C. (2017). Transformational Leadership and Organizational Processes: Influencing Public Performance. Public Administration Review, 77(4), 554-565. https://doi.org/10.1111/puar.12654

Tansky, J. W. \& Cohen, D. J. (2001). The relationship between organizational support, employee development, and organizational commitment: An empirical study. Human Resource Development Quarterly, 12(3), 285-300. https://doi.org/10.1002/hrdq.15 
Tourish, D. (2013). The Dark Side of Transformational Leadership. A critical pespective. London: Routledge.

Tracey, J. B. \& Hinkin, T. R. (1998). Transformational leadership or effective managerial practices? Group E Organization Management, 23(3), 220-236. https://doi.org/10.1177/1059601198233002

Veríssimo, J. \& Lacerda, T. (2015). Does integrity matter for CSR practice in organizations? The mediating role of transformational leadership. Business Ethics: A European Review, 24(1), 34-51. https://doi.org/10.1111/beer.12065

Vroom, V. H. \& Jago, A. G. (2007). The Role of the Situation in Leadership. American Psychologist, 62(1), 17-24. https://doi.org/10.1037/0003-066X.62.1.17

Yukl, G. A. (1999). An evaluation of conceptual weaknesses in transformational and charismatic leadership theories. Leadership Quarterly, 10(2), 285-305. https://doi.org/10.1016/S1048-9843(99)00013-2

Yukl, G. (2002). Leadership in organizations. 5 ed. New Jersey: Prentice Hall.

Wang, M. S. (2010). Influence of knowledge sharing and project complexity on group creativity: taking the development of information system for example. Journal of e-Business, 12(1), 73-102.

Wang, A. C. \& Cheng, B. S. (2010). When does benevolent leadership lead to creativity? The moderating role of creative role identity and job autonomy. Journal of Organizational Behavior, 31(1), 106-121. https://doi.org/10.1002/job.634

Wang, G., Oh, I. S., Courtright, S. H., \& Colbert, A. E. (2011). Transformational leadership and performance across criteria and levels: A meta-analytic review of 25 years of research. Group $\mathcal{E}$ Organization Management, 36(2), 223-270. https://doi.org/10.1177/1059601111401017

WAN-IFRA (2017, June 23). How newspaper brands survive the meltdown. Retrieved from https://blog.wan-ifra.org/2017/06/23/how-newspaper-brands-survive-the-meltdown-part-1

Zhang, X. \& Bartol, K. M. (2010). Linking empowering leadership and employee creativity: The influence of psychological empowerment, intrinsic motivation, and creative process engagement. Academy of management journal, 53(1), 107-128. https://doi.org/10.5465/AMJ.2010.48037118

Zhou, J. \& George, J. M. (2001). When job dissatisfaction leads to creativity: Encouraging the expression of voice. Academy of Management journal, 44(4), 682-696. https://doi.org/10.2307/3069410

Zwingmann, I., Wegge, J., Wolf, S., Rudolf, M., Schmidt, M., \& Richter, P. (2014). Is transformational leadership healthy for employees? A multilevel analysis in 16 nations. German Journal of Human Resource Management, 28(1-2), 24-51. https://doi.org/10.1688/ZfP-2014-01-Zwingmann

\section{SOBRE EL AUTOR}

Cristóbal Benavides, magister en Media Management y doctor en Comunicación por la Universidad de Navarra. Actualmente se desempeña como profesor asociado de la Facultad de Comunicación de la Universidad de los Andes e investigador responsable del proyecto Fondecyt 11150020 . Sus principales áreas de investigación son el liderazgo, la innovación en la industria de medios y el estudio de los usos y gratificaciones de los jóvenes en las diferentes plataformas. 